See discussions, stats, and author profiles for this publication at: https://www.researchgate.net/publication/336025399

\title{
Surgeon Training with Haptic Devices for Computer and Robot Assisted Surgery: An Experimental Study
}

Chapter · January 2020

DOI: 10.1007/978-3-030-31635-8_189

CITATIONS

0

5 authors, including:

Salih Ertug Ovur

Politecnico di Milano

4 PUBLICATIONS 2 CITATIONS

SEE PROFILE

Elena De Momi

Politecnico di Milano

204 PUBLICATIONS 1,464 CITATIONS

SEE PROFILE

Some of the authors of this publication are also working on these related projects:

Project EMG-Controlled Neuro-Prosthetic Devices View project

Project The Laryngeal dataset View project
READS

47

Marisa Cobanaj

Politecnico di Milano

1 PUBLICATION O CITATIONS

SEE PROFILE

Giancarlo Ferrigno

Politecnico di Milano

353 PUBLICATIONS 5,013 CITATIONS

SEE PROFILE 


\title{
Surgeon Training with Haptic Devices for Computer and Robot Assisted Surgery: An Experimental Study
}

\author{
Salih Ertug Ovur ${ }^{(\bowtie)}$, Marisa Cobanaj, Luca Vantadori, \\ Elena De Momi, and Giancarlo Ferrigno \\ Department of Electronics Informatics and Bioengineering, \\ Politecnico di Milano, Milan, Italy \\ salihertug.ovur@mail.polimi.it
}

\begin{abstract}
Development of robot-assisted minimally invasive surgery increasingly demands for efficient training methods. This paper describes an experiment exploring the use of haptic interaction for the purpose of skill training (haptic training). Various experiment tasks that include simple and complex tool paths have been developed for this purpose. 105 acquisition sessions distributed in 7 different tasks from 27 naive subjects and one surgeon performed teleoperated exercises with Omni Phantom. Task's learning curves with and without robotic assistance and effects of damping have been discussed. For the force guidance case, assistance level gradually decreased the applied force as the training progressed. In the controversial scenario of robotic assistance for motorlearning benefits, this study shows such assistance improve the rate of learning for both simple and complex tasks.
\end{abstract}

Keywords: Active constraints $\cdot$ Haptics $\cdot$ Surgical robotics training $\cdot$ Virtual-Reality $\cdot$ Augmented-Reality

\section{Introduction}

The advent of robot-assisted minimally invasive surgery (RAMIS), has the downside of a reduced, or, in the majority of the cases, completely absent sense of touch. Surgeons, therefore, have to rely on the visual cue to compensate for this lack and can exploit haptic training in order to improve the learning curve [1]. Haptics and computer-based Virtual Reality (VR) platforms together might play a key role in RAMIS to enhance the capabilities of the operator to improve the outcome of the surgical procedure in terms of safety, accuracy and cognitive load [2]. Substantial progress in the learning curve, indeed, has been achieved by using repetitive VR tasks before practicing on patients [3]. Moreover, computer-based VR training offers objective performance assessment during and after training able to accelerate the learning process $[4,5]$.

\footnotetext{
A brief video of this work is available on-line at: https://drive.google.com/open?id=1Qj92bBNK2JwA34MBKrPoXK0ZVNBqh6lg.
} 
To implement haptic feedback, systems for the intraoperative definition of active constraints have been developed. Latest researches about Active constraints focused to limiting the tool to within a certain space (forbidden region constraints) or attempt to guide the tool in moving along the desired path (guidance constraints) [6].

Benefits of the simulated environments in the case of forbidden region active constrain have been largely discussed and demonstrated [7, 8]. Force feedback systems for RAMIS typically measure or estimate the forces applied to the patient by the surgical instrument and generate a repulsive force on the robotic tool when it tries to enter the constrained, preventing common complication are accidental damages to nerves, veins and arteries [2].

Few works instead have found significant benefits of a simulated environment that enhance a training process with augmented forces [9], such as guidance constrain. The performance of attractive force applied to the robotic tool getting away from the constrained have been judged beneficial [10], null [11] and even detrimental [12].

\section{Related Works}

The guidance hypothesis [13] confirms that the guiding properties of augmented feedback are beneficial for motor learning when knowledge about the outcome is used to correct errors and improve subsequent performance, but detrimental when guiding affects processes important for learning. For example, practice with a high frequency of augmented feedback can affect learning by excessive reliance on external cues [12]. According to Schmidt [13], dependency on the robotic assistance can be addressed by gradually decreasing the guidance constraints intensity as the user learns. It provides the performer with an appropriate amount of haptic guidance throughout training [14]. This assistance-as-needed approach [15] prevents assistance from minimizing voluntary control, which has been shown to reduce the effectiveness of learning.

Moreover, haptic training supports passive learning, where subjects are not actively performing the task. In general, passively learning is not as efficient as actively one, committing errors and elaborating new strategies to accomplish the skill [16]. Therefore, haptic training strategies should involve the subjects' in actively participating and draw his attention to promote learning.

Finally, haptic guidance can considerably alter the dynamics of the task and affect motor learning. The guidance constraints can lead to unpredictable and unwanted motion of the tool. Indeed, the stored potential energy in common elastic links pulls the tool toward the desired path as long as the tool is away from the path. When the user relaxes his grasp on the haptic device, the stored energy can cause a sudden unintended motion of the tool and diminish the surgeon's overall control of the procedure. To overcome these issues, a solution has been proposed by [17]. A viscosity-based guidance active constraint is introduced: it attempts to guide the motion towards the desired path in the least aggressive way and without limiting the freedom of the user. Experimentation confirmed an improvement in accuracy of dynamic path following and a decrease in user's distraction. 
This paper presents an experimental study of haptic guidance support in training for teleoperated tasks. The work sums up solutions claimed by the mentioned experimental studies and aims to discuss such use of augmented forces for improving the training learning curve. Thus, it finally assesses the benefits of guidance constraints. For this purpose, the participants to the study have been trained in a VR environment with and without haptic feedback, and with damping force: their performance, during and after training, has been measured in order to enhance eventual improvements of subjects whose training has been assisted by guidance constraint.

\section{Materials}

\subsection{Omni Phantom Robot}

The PHANTOM Omni Touch Firewire was used for this research. Omni is six degreeof-freedom haptic manipulator with haptic feedback on the first three axes. Forward\&Inverse kinematics, haptic feedback controllers and damping parameters are implemented in $\mathrm{C}++.100 \mathrm{~Hz}$ is used for the haptic device updating rate. The tools' pose, forces, and task options were sent through common roscore to the laptop for visualization software.

\subsection{Visualization Software}

Visualization software has been realized in ROS (Robot Operating System) environment with $\mathrm{C}++$ code. The graphics are produced using the Visualization Toolkit (VTK) [18] and OpenGL [19] libraries with a refresh rate of $30 \mathrm{~Hz}$. Visualization software with VTK libraries makes possible to create various simulations for both VR and augmented reality (AR) environments.

\subsection{Experimental Setup}

Omni Phantom is integrated with control hardware and software that allows for reading the tool tip position and sending commands to the joints of the robot. Experimental setup while a participant surgeon is following tasks is given in Fig. 1.

The software architecture includes two main ROS-nodes: Omni phantom and visualization software. To prevent the computational load of the graphics from affecting the haptic control performance, these two were executed on separate computers: desktop computer running Ubuntu 14.04 LTS for haptic constraint control and laptop, featuring an Intel Core i7-7700HQ CPU and NVidia GTX 1050 GPU with Ubuntu 16.04 LTS, used for visualization software. 


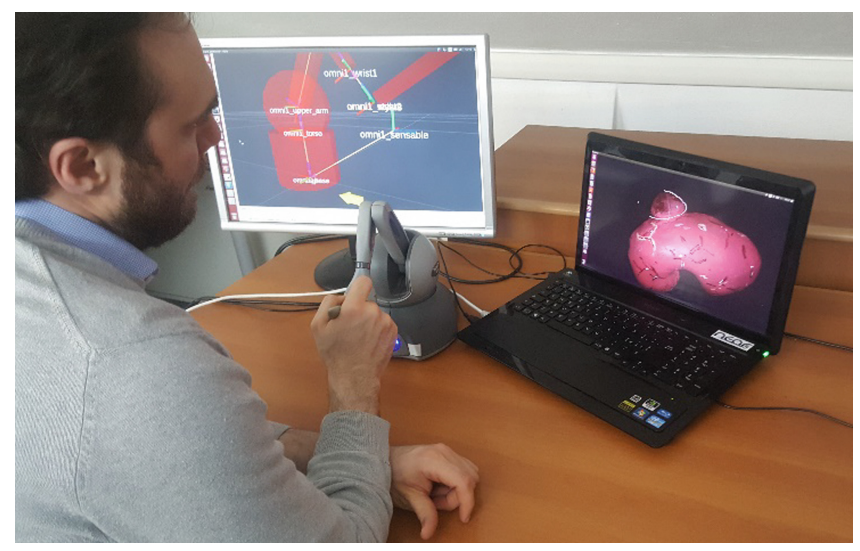

Fig. 1. The experimental setup

\section{Methods}

Designed tasks, acquisition procedure and finally, performance criteria of our study are introduced in this section.

\subsection{Tasks}

7 tasks are designed for simulating shapes in AR and VR but also with basic geometrical shapes such as rectangle, circle. Created shapes were followed by using Omni Phantom. All subjects trained on the same order of tasks (see Fig. 2): the contour of a kidney tumor on an AR scenario, a circle, a rectangle, an elliptical and an infinity shape, the contour of the renal artery during nephrectomy procedure on VR scenario and finally the first task again were performed. To keep subjects engaged and motivated intuitive performance color map feedback is provided through contour color shift from green to yellow and red, regarding the distance from the desired path Task 6 in VR has been introduced in order to make the subject dealing with virtual anatomy. Also, this more complicated shape could be employed to verify if guidance constraint on complicated paths can improve without the performance, as suggested in the literature.

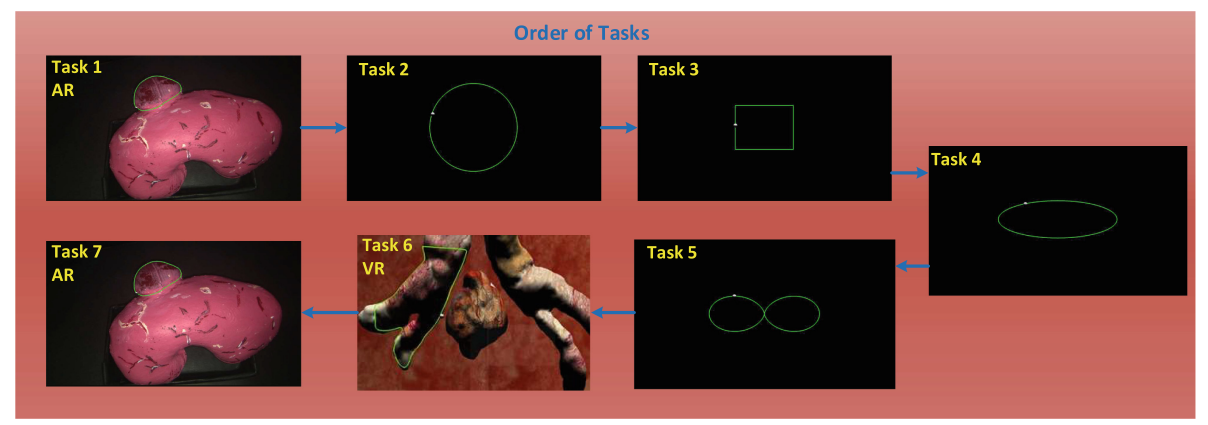

Fig. 2. Experiment structure design 
The shape contours of AR and VR tasks have been obtained from well-trained users. Filters have been applied in order to remove the effects of noise. All other shapes have been defined mathematically.

\subsection{Acquisition Procedure and Performance Metrics}

To enhance the effects of without the haptic guidance training, 27 non-medical participants (22 to 40 years old, 13 males and 14 females, all right-handed) were randomly divided into three groups of 9: a group that performed the tasks with no augmented force, a second one assisted by a damping effect and a third one with augmented force support for guidance towards the path. A medical participant instead accomplished all cases This way three groups of 10 participants each have been defined. Each group proceeded in sessions of 15 repetitions for each of the 7 tasks, lasting an average of $40 \mathrm{~min}$. Guidance force has been decreased so that at the end of the task no augmented force was applied: from a unitary value force, gain $\left(K_{o}\right.$ in Fig. 3$)$ was decreased each 3 repetitions by a factor 0.25 so that in the final 3 repetitions augmented force intensity was null. For the performance metrics, distance between cursor point and closest point on the followed path is computed.

\subsection{Robotic Assistance Method}

For the force assistance training, Fuzzy-PD controller has been used. It performs a control on the joint angle error, computed as the difference between the measured joint angles and desired joint angles:

$$
\theta_{\text {error }}=\theta_{\text {desired }}-\theta_{\text {measured }}
$$

Desired joint angles are obtained by using inverse kinematics on the desired path, as applied in [20]. $\theta_{\text {error }}$ and its derivative value $\dot{\theta}_{\text {error }}$ are used for computing the output $\left(\tau_{\text {controller }}\right)$, as seen in Fig. 4 . The controller Scaling Factors (SFs) $K_{e}$ and $K_{d}$ correspond respectively to the spring and damping constants. The input SFs together with saturation limits are used to normalize the inputs $\theta_{\text {error }}$ and $\dot{\theta}_{\text {error }}$ into the Membership Functions (MFs). The output $\mathrm{U}$ is converted into the control signal of the Fuzzy-PD as $\tau_{\text {controller }}=K_{o} U$ where $K_{o}$ is the output of SFs [21]. Tuned parameters for SFs: $\left(K_{e}=0.18, K_{d}=-1, K_{o}=2.2\right)$.

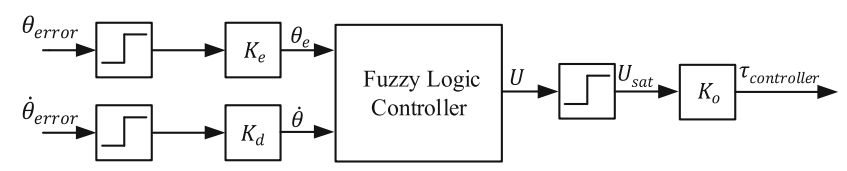

Fig. 3. General Scheme of the design 


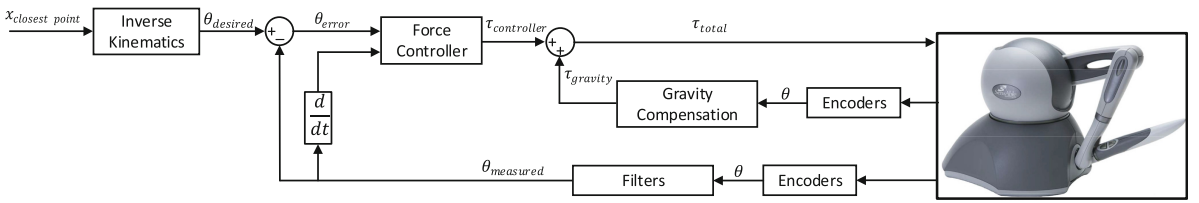

Fig. 4. Schematic joint space control diagram

The Fuzzy-PD is composed of a $5 \times 5$ rule base as given in Table 1 . The MFs are defined with triangular MFs and are defined with Negative Big (NB), Negative Medium (NM), Zero (Z), Positive Medium (PM) and Positive Big (PB) (Fig. 5). The consequent parts of the rules are defined with crisp singletons which are Positive Big $(P B=1)$, Positive Medium $(P M=0.7)$, Zero $(Z=0)$, Negative Medium $(\mathrm{NM}=-0.7)$ and Negative Big $(N B=-1)$. The resulting control surface is given in Fig. 6.

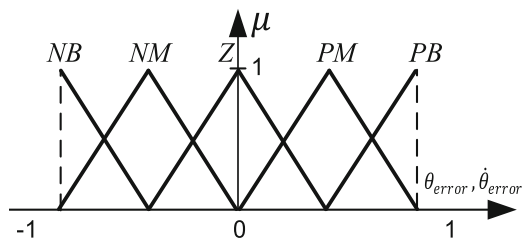

Fig. 5. Illustration of the MFs

Table 1. Rule base of Fuzzy-PD controller

\begin{tabular}{l|l|l|l|l|l}
\hline$X_{e} / \dot{X}$ & $\mathrm{~PB}$ & $\mathrm{PM}$ & $\mathrm{Z}$ & $\mathrm{NM}$ & $\mathrm{NB}$ \\
\hline $\mathrm{PB}$ & $\mathrm{PB}$ & $\mathrm{PB}$ & $\mathrm{PM}$ & $\mathrm{PM}$ & $\mathrm{Z}$ \\
\hline $\mathrm{PM}$ & $\mathrm{PB}$ & $\mathrm{PM}$ & $\mathrm{PM}$ & $\mathrm{Z}$ & $\mathrm{NM}$ \\
\hline $\mathrm{Z}$ & $\mathrm{PM}$ & $\mathrm{PM}$ & $\mathrm{Z}$ & $\mathrm{NM}$ & $\mathrm{NM}$ \\
\hline $\mathrm{NM}$ & $\mathrm{PM}$ & $\mathrm{Z}$ & $\mathrm{NM}$ & $\mathrm{NM}$ & $\mathrm{NB}$ \\
\hline $\mathrm{NB}$ & $\mathrm{Z}$ & $\mathrm{NM}$ & $\mathrm{NM}$ & $\mathrm{NB}$ & $\mathrm{NB}$ \\
\hline
\end{tabular}

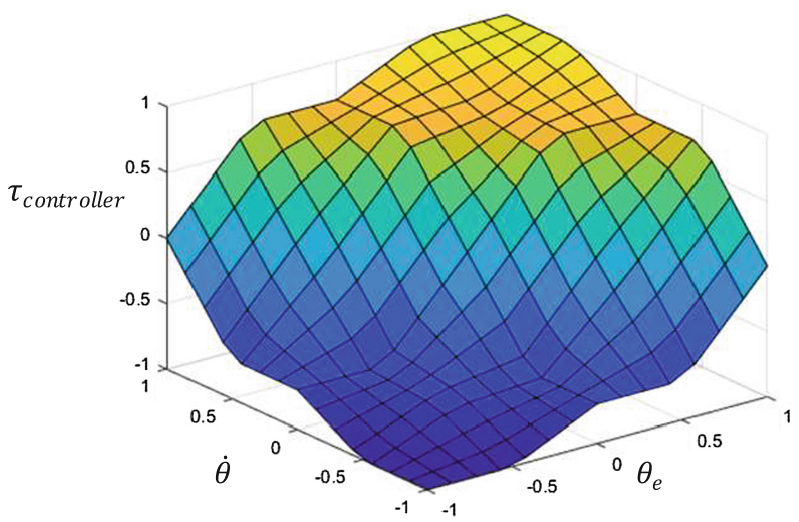

Fig. 6. Control surface of the Fuzzy-PD 
Gravity compensation provided for all experiments. Algorithm introduced at [22] is used for the compensation of gravity.

\section{Results and Discussion}

Results have been obtained for 3 different subject group, each one of 10 subjects (9 naive and the surgeon). Each participant performed for every 105 acquisitions, including 7 tasks and 15 repetitions for each task. During tasks, distance between cursor point from end effector of Omni and closest point on desired path are used for performance criteria. Error computed for each experiment has been computed according to the Root-Mean-Square method. Before proceeding, all obtained data have been normalized between maximum and minimum errors by excluding outliers (Normalized Error). Then, data have been collected for 10 people and their 7 tasks. This gives us distribution of error with respect to repetitions. For each repetition 70 data are considered. In order to comment on relations easier, performance criteria considered instead of an error. Maximum performance means minimum error and minimum performance equals maximum error. Obtained results are given in Fig. 7.

$$
\text { Performance Criteria }=1-\text { Normalized Error }
$$

In order to comment on obtained statistical results, normality tests are applied to learn if data present a normalized distribution. Firstly, one-sample KolmogorovSmirnov test has been applied on data for each repetition. As a result of the test, all data have a normal distribution $(\mathrm{p}<0.005)$ for each repetition $(\mathrm{N}=70)$. T-test and ShapiroWilk tests gave similar results. According to normally distributed data we could use mean and other main statistical parameters to comment on performance. However, we had also to check if there was a significant improvement between different subject groups. In order to find relations of different subject groups, t-test is used for studying relations of force enhanced, damping assisted and null subject groups (Table 2). Ttest's hypothesis is true $(h=1)$ when two sample's mean values are different.

Table 2. Statistical test results

\begin{tabular}{l|l|l}
\hline Compared subject groups & Hypothesis & $\mathrm{p}$-value (average) \\
\hline Force Assisted - Null & $\mathrm{h}=1$ for $(15 / 15$ repetitions $)$ & 0.005 \\
\hline Force Assisted - Damping Assisted & $\mathrm{h}=1$ for $(14 / 15$ repetitions $)$ & 0.01 \\
\hline Damping Assisted - Null & $\mathrm{h}=0$ for $(15 / 15$ repetitions) & 0.6 \\
\hline
\end{tabular}

Results of these tests between subject groups show that there is a meaningful difference between force assisted group with respect to null and damping assisted groups. However, there is no guaranteed distinction between damping assisted with respect to null subject groups. Moreover, these relations are also verified with Bonferroni Correction. For the analytical comparison, only the last 3 repetitions have been considered, where force-assistance is totally removed. For the comparison, mean and \% 95 Confidence Interval (CI) has been used (see Table 3). 

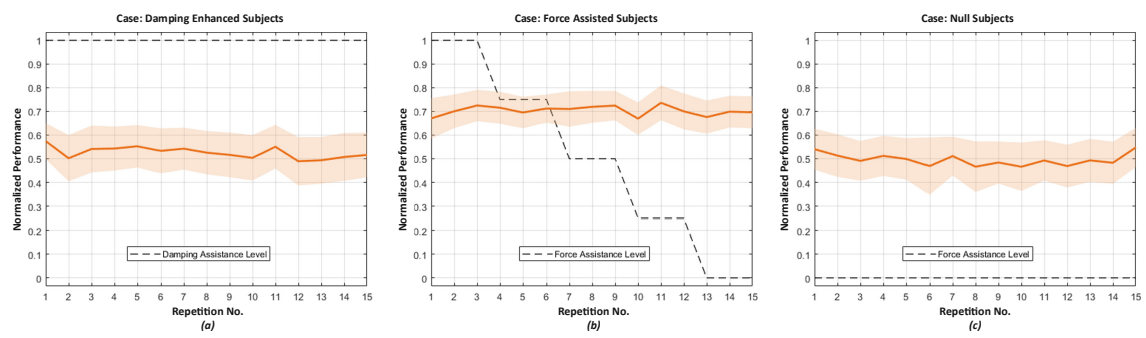

Fig. 7. The performance analysis of (a) damping enhanced, (b) force assisted and (c) null subjects. Dark orange line shows the mean and light-orange area represents the $\% 95$ confidence interval for 7 tasks and 10 people for each repetition.

Table 3. $\mu$ and CI of all groups performance

\begin{tabular}{|c|c|c|c|c|c|c|}
\hline \multirow[t]{2}{*}{ Repetition no. } & \multicolumn{2}{|c|}{ Damping asst. } & \multicolumn{2}{|c|}{ Force asst. } & \multicolumn{2}{|l|}{ Null } \\
\hline & $\mu$ & CI (\%95) & $\mu$ & CI (\%95) & $\mu$ & CI (\%95) \\
\hline \multirow[t]{2}{*}{13} & \multirow[t]{2}{*}{0.49} & 0.59 & \multirow[t]{2}{*}{0.68} & 0.76 & \multirow[t]{2}{*}{0.49} & 0.58 \\
\hline & & 0.39 & & 0.63 & & 0.40 \\
\hline \multirow[t]{2}{*}{14} & \multirow[t]{2}{*}{0.51} & 0.61 & \multirow[t]{2}{*}{0.70} & 0.77 & \multirow[t]{2}{*}{0.48} & 0.57 \\
\hline & & 041 & & 0.63 & & 0.39 \\
\hline \multirow[t]{2}{*}{15} & \multirow[t]{2}{*}{0.52} & 0.61 & \multirow[t]{2}{*}{0.70} & 0.75 & \multirow[t]{2}{*}{0.55} & 0.63 \\
\hline & & 0.42 & & 0.61 & & 0.46 \\
\hline
\end{tabular}

Figure 7 visually shows, and Table 3 statistically proves us there are significant improvement occurred for the force assisted subjects. Although force assistance level reduced repetitions show decrease in performance, subjects could tolerate the changes and they could keep the same level of performance. Moreover, the normalized error has been compared for different tasks performed by the same subject and it was noticed that for all subjects, the highest improvement in performance occurs for the most complex task (Task 6). This confirms what supported by the state-of-art. However, damping assistance did not significantly increase performance which could be caused by, damping was only applied for only the first three joints.

\section{Conclusions}

This work proposed a haptic guidance method in surgical robotic training. According to the literature findings and our conclusion, applied haptic guidance during training increases the performance. The method was put to test through a comparison with a no force and damping, whose efficacy has been supported in some studies we wanted to validate, assisted training. 30 experiments sessions, in which 27 novice participants and a surgeon followed 7 different tasks with 15 repetitions for each of them, were considered. Results confirmed that after the force removal, the performance of subjects with haptic guidance training kept at a higher rate during all experiments with respect 
to one of the subjects with no force and damping supported training. Therefore, it is possible to assess that in this study the guidance constraints have a beneficial effect on improving the training learning curve. These results are promising, and further investigation is needed to validate this work with different tasks. The study was performed on a relatively small sample due to the difficulties in recruiting subjects for a quite long experiment and a larger sample might lead to more significant results.

Acknowledgment. We received assistance on the software from Hang Su. We thank our subjects for kindly participating in the training experiment. This work was supported by European Union's Horizon 2020 research and innovation programme (grant agreement No. 732515 "SMARTsurg").

\section{References}

1. Wagner, C.R., Stylopoulos, N., Jackson, P.G., Howe, R.D.: The benefit of force feedback in surgery: examination of blunt dissection. Presence Teleoperators Virtual Environ. 16, 252 262 (2007)

2. Vantadori, L., Mariani, A., Chupin, T., De Momi, E., Ferrigno, G.: Design and evaluation of an intraoperative safety constraints definition and enforcement system for robot-assisted minimally invasive surgery. In: Proceedings of Computer/Robot Assisted Surgery CRAS (2018)

3. Enayati, N., De Momi, E., Ferrigno, G.: Haptics in robot-assisted surgery: challenges and benefits. IEEE Rev. Biomed. Eng. 9, 49-65 (2016)

4. Sanchez, B.R., Mohr, C.J., Morton, J.M., et al.: Comparison of totally robotic laparoscopic Roux-en-Y gastric bypass and traditional laparoscopic Roux-en-Y gastric bypass. Obes. Surg. 16, 690-696 (2006)

5. Seymour, N.E., Gallagher, A.G., Roman, S.A., et al.: Virtual reality training improves operating room performance: results of a randomized, double-blinded study. Annu. Surg. 6, 458-463 (2002)

6. Lau, J.W., Yang, T., Toe, K.K., Huang, W., Chang, S.K.: Can robots accelerate the learning curve for surgical training? An analysis of residents and medical students. Ann. Acad. Med. Singap. 1, 29-35 (2018)

7. Okamura, A.: Haptic feedback in robot-assisted minimally invasive surgery. Curr. Opinion Urol. 19, 102-107 (2009)

8. Bowyer, S.A., et al.: Active constraints/virtual fixtures: a survey. Trans. Robot. 30, 138-157 (2014)

9. Sigrist, R., Rauter, G., Riener, R., Wolf, P.: Augmented visual, auditory, haptic, and multimodal feedback in motor learning: a review. Psychon. Bull. Rev. 20, 21-53 (2013)

10. Enayati, N., Okamura, A.M., Mariani, A., Pellegrini, E., Coad, M.M., Ferrigno, G., De Momi, E.: Robotic assistance-as-needed for enhanced visuomotor learning in surgical robotics training: an experimental study. In: IEEE International Conference on Robotics and Automation (ICRA) (2018)

11. Jacobs, S., Holzhey, D., Strauss, G., Oliver Burgert, O., Falk, V.: The impact of haptic learning in telemanipulator-assisted. Surg. Laparosc. Endosc. Percutaneous Tech. 17, 402 406 (2007)

12. Coad, M.M., et al.: Training in divergent and convergent force fields during 6-DOF teleoperation with a robot-assisted surgical system. In: IEEE World Haptics Conference (WHC) (2017) 
13. Schmidt, R.A.: Frequent augmented feedback can degrade learning: evidence and interpretations. In: Tutorials in Motor Neuroscience, vol. 62, pp. 59-75. Springer, Dordrecht (1991)

14. Li, Y., Huegel, J.C., Patoglu, V., O’Malley, M.K.: Progressive shared control for training in virtual environments. In: 3rd Joint EuroHaptics Conference and Symposium on Haptic Interfaces for Virtual Environment and Teleoperator Systems. World Haptics, pp. 332-337 (2009)

15. Marteniuk, R.G.: Retention characteristics of motor short-term memory cues. J. Motor Behav. 5, 249-257 (1973)

16. Crespo, L.M., Reinkensmeyer, D.J.: Haptic guidance can enhance motor learning of a steering task. J. Motor Behav. 40, 545-557 (2014)

17. Enayati, N., Alves Costa, E.C., Ferrigno, G., De Momi, E.: A dynamic non-energy-storing guidance constraint with motion redirection for robot-assisted surgery. In: IEEE International Conference on Intelligent Robots and Systems (IROS), Daejeon Convention Center, Korea (2016)

18. VTK Homepage. https://vtk.org/. Accessed 26 Apr 2019

19. OpenGL Resources page. https://www.opengl.org/resources/. Accessed 26 Apr 2019

20. Gudino-Lau, J., Chavez-Montejano, F., Alcala, J., Charre-Ibarra, S.: Direct and inverse kinematic model of the OMNI PHANToM. ECORFAN-Bolivia J. 5, 25-32 (2018)

21. Hong, M., Rozenblit, J.W.: Haptic guidance with fuzzy control in simulation. In: Proceedings of the Symposium on Modeling and Simulation in Medicine, Alexandria, Virginia, pp. 25-30 (2015)

22. Koul, M.H., Kumar, P., Singh, P.K., Manivannan, M., Saha, S.K.: Gravity compensation for PHANToMTM Omni ${ }^{\circledR}$ haptic interface. In: First Joint International Conference on Multibody System Dynamics (2015) 